\title{
New horizons in the regulation of bile acid and lipid homeostasis: critical role of the nuclear receptor FXR as an intracellular bile acid sensor
}

Sinal CJ, Tohkin M, Miyata $M$, et al. Targeted disruption of the nuclear receptor FXR/BAR impairs bile acid and lipid homeostasis. Cell 2000;102:731-44.

\section{Abstract}

Mice lacking the nuclear bile acid receptor FXR/BAR developed normally and were outwardly identical to wild-type littermates. FXR/BAR null mice were distinguished from wild-type mice by elevated serum bile acid, cholesterol, and triglycerides, increased hepatic cholesterol and triglycerides, and a proatherogenic serum lipoprotein profile. FXR/BAR null mice also had reduced bile acid pools and reduced fecal bile acid excretion due to decreased expression of the major hepatic canalicular bile acid transport protein. Bile acid repression and induction of cholesterol 7alphahydroxylase and the ileal bile acid binding protein, respectively, did not occur in FXR/BAR null mice, establishing the regulatory role of FXR/BAR for the expression of these genes in vivo. These data demonstrate that FXR/BAR is critical for bile acid and lipid homeostasis by virtue of its role as an intracellular bile acid sensor.

\section{Comment}

Bile acids (BA) play an important role in human physiology. ${ }^{1}$ As amphipathic water soluble end products of cholesterol metabolism, they participate in body cholesterol disposal as well as generation of bile flow and biliary lipid secretion. However, in spite of being key endobiotics, BA are intrinsically toxic for cells mainly because of their inherent detergent and membrane disruptive properties. In fact, BA induced hepatotoxicity has been implicated in the pathogenesis and perpetuation of liver injury in cholestatic liver diseases. ${ }^{2}$ Therefore, it is not surprising that intracellular BA levels are tightly maintained within a narrow concentration range ${ }^{3}$ as too much leads to hepatotoxicity while too little can lead to significant impairments in bile flow and luminal fat digestion. How the liver orchestrates the regulation of intracellular BA concentrations appears to be through transcriptional regulation of genes involved in both BA biosynthesis and transport. The recent cloning and identification of major enzymes and transport proteins involved in the synthesis and enterohepatic circulation of BA have provided insight into how this regulation takes place. ${ }^{45}$ Available data suggest that BA may indeed participate in transcriptional activation or repression of several genes involved in the conversion of cholesterol into BA as well as hepatic transport. ${ }^{6}$ Relevant examples are BA mediated feedback inhibition of cholesterol 7alpha-hydroxylase (CYP7A1), ${ }^{7}$ the rate limiting enzyme in BA biosynthesis, and the sinusoidal $\mathrm{Na}(+) /$ bile acid cotransporter (Ntcp), the main uptake system for BA in the liver. ${ }^{8}$ Several recent reports have shed light on the mechanisms mediating BA regulation of target genes. ${ }^{9}{ }^{10}$

Building on the recent discovery that FXR is a BA activated nuclear receptor that can regulate expression of $\mathrm{BA}$ responsive target genes in the intestine and liver, ${ }^{11}{ }^{12}$ the paper by Sinal et al explores the effects of FXR deficiency in vivo and therefore provides an important physiological setting for the central role of FXR in controlling BA homeostasis. Using now standard genetic ablation techniques, FXR -/- mice were generated. Knockout mice were viable, fertile, grew normally, and exhibited elevated serum levels of BA, cholesterol, and triglycerides, increased hepatic cholesterol and triglyceride levels, a reduced bile acid pool size, and decreased faecal bile acid excretion. This phenotype indicates that defective hepatic uptake and excretion of BA are present in FXR -/- mice and that FXR has a profound influence on lipid metabolism. In fact, when the authors assessed expression of genes involved in hepatic and ileal BA transport as well as genes controlling BA biosynthesis, they found a significant decrease in mRNA levels of the major BA transporter at the canalicular membrane, the bile salt export pump (Bsep) and the putative intracellular intestinal transporter of bile acids (the ileal bile acid binding protein) while CYP7A1 message was increased. Together, these data indicate that under basal conditions, FXR is required for normal expression of these genes. To further study the influence of FXR on BA and lipid homeostasis, Sinal et al fed control and FXR -/mice with either BA ( $1 \%$ cholic acid) or cholesterol enriched chow. On dietary challenge with cholic acid, FXR -/- mice developed severe hepatotoxicity with marked vacuolation and necrosis of liver cells on histological analysis reflecting an inability of the knockout mice to deal with BA overload. In contrast, normal mice tolerated well administration of cholic acid and did not show overt signs of toxicity. When expression of genes involved in BA metabolism was analysed, normal mice responded to BA feeding with downregulation of CYP7A1 and Ntcp mRNA levels, and upregulation of Bsep mRNA, while null animals were unable to display these changes in gene expression. These findings suggest that the regulatory response of hepatocytes to BA overload consists of reducing BA synthesis and import, and increasing BA export. These data indicate that FXR plays a fundamental role in protecting the liver against pathophysiological levels of BA. In addition, Sinal et al observed that FXR deficiency is associated with a significant reduction in mRNA levels of the BA activated transcriptional repressor SHP (small heterodimer partner) which is known to mediate CYP7A1 and Ntcp downregulation. ${ }^{8}$ On BA feeding of wild-type mice, SHP expression was markedly upregulated while FXR -/mice were again unable to display this response. This observation confirms that SHP expression is under direct control of FXR. ${ }^{10}$ 
Cholesterol feeding of FXR -/- mice resulted in intrahepatic lipid accumulation and hepatomegaly. Moreover, the proatherogenic lipoprotein profile of FXR -/- fed normal chow was exacerbated by cholesterol feeding as null mice accumulated low density lipoprotein and apo-E containing high density lipoprotein. The underlying mechanisms of this response are not clear from experimental data. Excess cholesterol can be converted into oxysterols signalling molecules which modulate the activity of a number of transcription factors, so as to limit accumulation of excess cholesterol. In view of the essential role of nuclear receptors as regulators of cholesterol catabolism, it is conceivable that crosstalk between them might serve to regulate lipid homeostasis. ${ }^{13}$ Sinal et al hypothesize that hepatic lipid accumulation seen in FXR null animals probably represents an indirect effect of impaired BA excretion which is known to be closely connected to biliary lipid secretion. Unfortunately, no determinations of biliary solutes were carried out in the knockout mice.

The work of Sinal et al provides exciting new data and generates insights into bile physiology. FXR -/- mice represent valuable experimental tools to assess several aspects of cholestatic liver injury as well as the biological effects of $\mathrm{BA}$ at the molecular level. They will also allow studies on BA toxicity and the participation of FXR and other nuclear receptors, such as the pregnane $\mathrm{X}$ receptor, ${ }^{14}{ }^{15}$ in $\mathrm{BA}$ detoxification.

In summary, the paper of Sinal et al demonstrated that FXR is critical as an endogenous BA receptor and is a major regulator of bile acid homeostasis. Therefore, it is conceivable that altered expression or regulation of FXR may play a role in several diseases related to bile acid metabolism or transport. In addition, as FXR seems to be critical for preventing intracellular accumulation of toxic concentrations of BA, it constitutes a potential molecular target that can be modulated as a novel therapeutic strategy for cholestasis and other hepatic diseases.
Department of Gastroenterology,

M ARRESE

Catholic University of Chile School of Medicine,

Santiago, Chile

Texas Children's Liver Center, Department of Pediatrics,

S J KARPEN

Baylor College of Medicine, Texas, USA

Correspondence to: Dr M Arrese, Department of Gastroenterology, Catholic University of Chile School of Medicine, Marcoleta No 347, Santiago, Postal code 6510260, Chile.

marrese@med.puc.cl

1 Hofmann AF. The continuing importance of bile acids in liver and intestinal disease. Arch Intern Med 1999;159:2647-58.

2 Sodeman T, Bronk SF, Roberts PJ, et al. Bile salts mediate hepatocyte apoptosis by increasing cell surface trafficking of Fas. Am $\mathcal{f}$ Physiol Gastrointest tosis by increasing cell surface traf
Liver Physiol 2000;278: G992-9.

3 Setchell KD, Rodrigues CM, Clerici C, et al. Bile acid concentrations in human and rat liver tissue and in hepatocyte nuclei. Gastroenterology 1997; 112:226-35

4 Vlahcevic ZR, Pandak WM, Stravitz RT. Regulation of bile acid biosynthesis. Gastroenterol Clin North Am 1999;28:1-25.

5 Kullak-Ublick GA, Stieger B, Hagenbuch B, et al. Hepatic transport of bile salts. Semin Liver Dis 2000;20:273-92.

6 Muller M. Transcriptional control of hepatocanalicular transporter gene expression. Semin Liver Dis 2000;20:323-37.

7 Stroup D, Crestani M, Chiang JY. Identification of a bile acid response element in the cholesterol 7 alpha-hydroxylase gene CYP7A. Am f Physiol Gastrointest Liver Physiol 1997;273:G508-17.

8 Denson LJ, Sturm E, Echeverría W, et al. Bile acid-mediated feedback inhibition of the Ntcp promoter gene occurs via a novel mechanism involving the bile acid receptor FXR activation of a transcriptional receptor. Gastroenterology 2001;121:140-7.

9 Lu TT, Makishima M, Repa JJ, et al. Molecular basis for feedback regulation Lu TT, Makishima M, Repa JJ, et al. Molecular basis for feedback reg
of bile acid synthesis by nuclear receptors. Mol Cell 2000;6:507-15.

10 Goodwin B, Jones SA, Price RR, et al. A regulatory cascade of the nuclear receptors FXR, SHP-1, and LRH-1 represses bile acid biosynthesis. Mol Cell 2000;6:517-26.

11 Makishima M, Okamoto AY, Repa JJ, et al. Identification of a nuclear receptor for bile acids. Science 1999;284:362-5.

12 Parks DJ, Blanchard SG, Bledsoe RK, et al. Bile acids: natural ligands for an orphan nuclear receptor. Science 1999;284:1365-8.

13 Chawla A, Saez E, Evans RM. Don't know much bile-ology. Cell 2000;103: $1-4$

14 Xie W, Radominska-Pandya A, Shi Y, et al. An essential role for nuclear receptors SXR/PXR in detoxification of cholestatic bile acids. Proc Natl Acad Sci USA 2001;98:3375-80.

15 Staudinger JL, Goodwin B, Jones SA, et al. The nuclear receptor PXR is a lithocholic acid sensor that protects against liver toxicity. Proc Natl Acad Sci USA 2001;98:3369-74. 